\title{
CONFIABILIDADE DE UM MÉTODO DE ANÁLISE HISTOLÓGICA DA DEGENERAÇÃO DISCAL EXPERIMENTAL EM COELHOS
}

\author{
RELIABILITY OF A METHOD FOR HISTOLOGICAL ANALYSIS OF EXPERIMENTAL \\ DISC DEGENERATION IN RABBITS \\ CONFIABILIDAD DE UN MÉTODO PARA EL ANÁLISIS HISTOLÓGICO DE LA \\ DEGENERACIÓN DISCAL EXPERIMENTAL EN CONEJOS
}

Emiliano Neves Vialle ${ }^{1}$ Luiz Roberto Gomes Vialle ${ }^{1}$, Antônio Bernardo de Queiroz Krieger², André de Oliveira Arruda ${ }^{3}$, Ricardo Nascimento Riet ${ }^{3}$, Misael Gomes Barbosa ${ }^{4}$, Lucia de Noronha ${ }^{5}$

\begin{abstract}
RESUMO
Objetivo: Validar um método para avaliação histológica da degeneração discal experimental em coelhos, baseando-se na confiabilidade da análise interobservador. Métodos: Treze coelhos da raça New Zealand foram submetidos a procedimento de indução da degeneração discal através de punção direta de três discos intervertebrais (DIV) consecutivos com agulha 18G sob anestesia. Ao fim de dois meses, coletou-se a coluna vertebral completa de cada coelho e procedeu-se preparo das peças para análise histológica dos discos intervertebrais (Experimentais e Controle), sendo coradas pelo método hematoxilina-eosina. As lâminas histológicas foram avaliadas quanto à ocorrência de degeneração através da análise dos seguintes critérios: presença de vasos sanguíneos; presença de protrusão do núcleo pulposo (NP) através do ânulo fibroso (AF); e ruptura das fibras do AF. Resultados: Ao final dos processos de eutanásia, retirada da coluna e preparo histológico, obteve-se 60 DIV viáveis para avaliação de degeneração. Deste total, 25 peças eram de DIV experimentais e 35 peças de DIV controle. A presença de vaso sanguíneo foi observada em 18 dos 25 DIV degenerados, com concordância de Kappa = 0,95 entre os observadores. A presença da extrusão do NP foi identificada em 19 dos 25 DIV experimentais, com concordância de Kappa $=0,78$ entre os observadores. Com relação à ruptura das fibras do AF, pode-se identificar a positividade em 24 dos 25 DIV degenerados, com concordância entre os observadores de Kappa =0,65. Conclusão: Este modelo de avaliação histológica da degeneração experimental do DIV mostrou-se viável, com alto grau de concordância entre os observadores na identificação da degeneração discal.
\end{abstract}

Descritores: Degeneração do disco intervertebral; Histologia; Modelos animais; Coelhos; Reprodutibilidade dos testes.

\begin{abstract}
Objective: To validate a method for histological evaluation of disc degeneration in rabbits based on the reliability of interobserver analysis. Methods: Thirteen New Zealand white rabbits underwent a procedure to induce disc degeneration through direct puncture of three consecutive intervertebral discs (IVD) with an 18G needle under anesthesia. After two months, the complete spine of each rabbit was collected, and further prepared for histologic examination of the intervertebral discs (Experimental and Control), and stained with the hematoxylin-eosin method. Histology slides were evaluated for the occurrence of degeneration by analyzing the following criteria: presence of blood vessel, presence of protrusion of the nucleus pulposus (NP) through the annulus fibrosus (AF), and rupture of the fibers of the AF. Results: After the euthanasia procedures, collection of the spine and histological preparation, 60 viable IVD were obtained for degeneration assessment. Of a total of 60 pieces, 25 pieces were from Experimental IVD and 35 pieces were from Control IVD. The presence of blood vessel was observed in 18 of 25 degenerated IVD with agreement among observers of Kappa $=0.95$. The presence of the extrusion of the NP was identified in 19 of 25 experimental IVD with interobserver agreement of Kappa $=0.78$. The rupture of the fibers of AF could be identified in 24 of 25 degenerated IVD, with interobserver agreement of Kappa $=0.65$. Conclusion: This model of histological assessment of experimental IVD degeneration is feasible, with a high degree of interobserver agreement in the identification of disc degeneration.
\end{abstract}

Keywords: Intervertebral disc degeneration; Histology; Animal models; Rabbits; Reproducibility of results.

\section{RESUMEN}

Objetivo: Validar un método para evaluación histológica de la degeneración discal experimental en conejos, basándose en la confiabilidad del análisis interobservadores. Métodos: Trece conejos de la raza New Zealand fueron sometidos a procedimiento de inducción de la degeneración discal a través de punción directa de tres discos intervertebrales (DIV) consecutivos con aguja 18G bajo anestesia. Al fin de dos meses, se colectó la columna vertebral completa de cada conejo y se procedió a la preparación de las piezas para análisis histológico de los discos intervertebrales (Experimentales y Controles), siendo coloreadas por el método hematoxilina-eosina. Las láminas histológicas fueron evaluadas cuanto a ocurrencia de degeneración a través del análisis de los siguientes criterios: presencia de vasos sanguíneos; presencia de protrusión del núcleo pulposo (NP) a través del anillo fibroso (AF); y rotura de las fibras del AF. Resultados:

1. Médicos do Grupo de Cirurgia de Coluna, Hospital Universitário Cajuru, Pontifícia Universidade Católica do Paraná, Paraná, Brasil.

2. Médico Estágiário do Grupo de Cirurgia de Coluna, Hospital Universitário Cajuru, Mestrando em Cirurgia da Pontifícia Universidade Católica do Paraná, Paraná, Brasil.

3. Aluno do Curso de Medicina, Pontifícia Universidade Católica do Paraná, Paraná, Brasil.

4. Aluno do Curso de Biologia, Pontifícia Universidade Católica do Paraná, Paraná, Brasil.

5. Professora Adjunta de Anatomia Patológica, Pontifícia Universidade Católica do Paraná, Paraná, Brasil.

Trabalho realizado na Pontifícia Universidade Católica do Paraná - PUC-PR.

Correspondência: Rua Brigadeiro Franco, 979.CEP 80430-210, Curitiba, PR, Brasil. Email: emiliano @ vialle.com.br

Recebido em 28/04/2010, aceito em 16/08/2010. 
Al final de los procesos de eutanasia, remoción y preparación histológica de la columna, se obtuvieron 60 DIV viables para evaluación de degeneración. De este total, 25 piezas eran de DIV experimentales y 35 piezas de DIV control. La presencia de vaso sanguíneo fue observada en 18 de los 25 DIV degenerados, con concordancia de Kappa = 0,95 entre los observadores. La presencia de la extrusión del NP fue identificada en 19 de los 25 DIV experimentales, con concordancia de Kappa = 0,78 entre los observadores. Con relación a la rotura de las fibras del AF, se puede identificar la positividad en 24 de los 25 DIV degenerados, con concordancia entre los observadores de Kappa = 0,65. Conclusión: Este modelo de evaluación histológica de la degeneración experimental del DIV se mostró viable, con alto grado de concordancia entre los observadores en la identificación de la degeneración discal.

Descriptores: Degeneración del disco intervertebral; Histología; Modelos animales; Conejos; Reproductibilidad de los tests.

\section{INTRODUÇÃO}

A degeneração discal é uma afecção que compreende a perda das características estruturais, biológicas e bioquímicas do disco intervertebral ${ }^{1}$, caracterizando-se por uma importante perda da rede de proteoglicanos e água do núcleo discal². Isso pode levar à evidência clínica de degeneração - razão do decréscimo da altura discal - acarretando em aumento de carga nas estruturas próximas à coluna e, finalmente, alterando as suas relações mecânicas ${ }^{3}$. Histologicamente ocorre aumento da proliferação celular com formação de aglomerados, e, paradoxalmente, intensificação da morte de células - tanto pelo processo de apoptose quanto pela necrose tecidual ${ }^{4}$. Além disso, observa-se desorganização lamelar, fissuras e aumento da vascularização local ${ }^{5}$. Alterações moleculares também são encontradas, como um aumento na produção de citocinas e enzimas destruidoras de matriz (metaloproteinases), além de interleucina-6, óxido nítrico e prostaglandina E2 ${ }^{6}$, e um decréscimo dos seus componentes intracelulares (proteoglicanos), levando à perda gradual de fluido intradiscal ${ }^{7}$.

Boos et al. ${ }^{8}$ descreveram em detalhe as alterações progressivas na histologia do disco que ocorrem com a idade. Estas mudanças incluem um aumento no número fissuras, ruptura de fibras, presença de material granular, e neovascularização de fora para dentro do núcleo. Proliferação celular, formação de aglomerados celulares, acentuação da morte celular, perda da demarcação entre o ânulo e o núcleo também aumentam com a idade. Além disso, observam-se desorganizações estruturais da placa terminal, que incluem fissuras, afilamento da cartilagem, alteração da densidade celular, microfraturas do osso subcondral adjacente e esclerose óssea ${ }^{8}$.

Em termos de epidemiologia e consequências sociais, um dos sintomas mais importantes da degeneração discal corresponde à dor lombar crônica, chamada Degeneração Discal Sintomática (DDS). Esta é a segunda maior causa de consulta médica nos Estados Unidos ${ }^{9-11}$, sendo gastos, segundo dados americanos, 20 bilhões de dólares ao ano em custos diretos para tratamento da dor lombar crônica que, somados às despesas indiretas, corresponde a um valor que ultrapassa os 100 bilhões de dólares ${ }^{12}$.

A fim de amenizar esse quadro alarmante, diversos modelos biológicos de tratamento têm sido propostos, para futuras aplicações de novas tecnologias. Há uma evidente dificuldade de se obter modelo similar ao humano, tanto por questões éticas, quanto por diferenças anatômicas, fisiológicas e histológicas entre as variadas espécies que estão ao alcance da pesquisa experimental. Mesmo após diversos estudos ${ }^{13-16}$ experimentais, não existe consenso entre os autores quanto ao melhor método de análise da degeneração discal em modelo animal.

Sendo assim, o objetivo deste estudo é validar um método para avaliação histológica da degeneração discal experimental em coelhos, baseando-se na confiabilidade da análise inter-observador.

\section{MÉTODOS}

Este estudo foi avaliado e aprovado pelo Comitê de Ética no Uso de Animais da Pontifícia Universidade Católica do Paraná sob o número 252, parecer 180/07, sendo executado de acordo com as normas da Declaração de Helsinki da Associação Médica Mundial.

\section{Cirurgia para indução de degeneração discal}

Este estudo utilizou 13 coelhos da raça New Zealand, machos, pesando entre 3,5 e 4,5kg, com idade média de um ano. Cada um dos animais foi submetido a procedimento de indução da degeneração discal através de três punçõesão diretas do disco com agulha $18 \mathrm{G}$ sob anestesia, anteriormente validado por estes autores ${ }^{17}$.

Após indução anestésica com administração intramuscular de cloridrato de ketamina $5 \%(25 \mathrm{mg} / \mathrm{kg})$ e xilazina $(0,15 \mathrm{mg} / \mathrm{kg})$, os coelhos foram posicionados em decúbito lateral direito, tendo as superfícies laterais de três DIV lombares - L2-L3, L3-L4, L4-L5 (DIV Experimentais) expostas através de uma abordagem posterolateral retroperitoneal. O local da punção era cuidadosamente identificado para, em seguida, ser puncionado por agulha 18G moldada em forma de baioneta para padronização da profundidade de perfuração em $5 \mathrm{~mm}$. Os demais DIV lombares que não foram submetidos à indução da degeneração foram discriminados como DIV Controle.

\section{Preparo histológico}

Ao fim de dois meses de acompanhamento da evolução dos animais, procedeu-se a eutanásia através de injeção de dose letal de pentobarbital $(90 \mathrm{mg} / \mathrm{kg}$ ). Em seguida, coletou-se a coluna vertebral completa, sendo mantida em solução de formol 10\% pelo período de cinco dias. Após, procedeu-se a limpeza manual das peças anatômicas, preservando-se apenas as estruturas ósseas e cartilaginosas, que foram colocadas em solução descalcificante (ácido etilenodiamino tetra-acético - EDTA) por cerca de dois meses. Cada coluna foi divida em conjuntos compostos por um DIV e suas placas vertebrais superior e inferior correspondentes, permanecendo durante mais uma semana em EDTA para finalização do processo. Ao fim deste período, cada conjunto foi amostrado através de cortes transversais manuais com lâmina de micrótomo, permitindo a observação histológica de cada um dos DIV Experimentais, DIV Controle, bem como de suas placas vertebrais. Após a amostragem, o material foi submetido à desidratação, diafanização e impregnação em parafina com histotécnico Leica ${ }^{\circledR}$ modelo EG1160, utilizando-se o inclusor Leica ${ }^{\circledR}$ EG1160. Os blocos foram cortados com micrótomo (Leica ${ }^{\circledR}$ RM2145) na espessura de $5 \mu \mathrm{m}$, gerando um total de 120 cortes histológicos (DIV e placas). Finalizado o preparo, o material foi disposto em lâmina de vidro com albumina, sendo corados pelo método hematoxilina-eosina (HE), conforme protocolo de preparo histológico empregado pelo Laboratório de Patologia Experimental da Pontifícia Universidade Católica do Paraná. Para este estudo utilizamos apenas os cortes corados com HE. 


\section{Avaliação histológica}

As lâminas histológicas de DIV obtidas foram avaliadas quanto à ocorrência de degeneração através da análise dos seguintes critérios: presença de vaso sanguíneo; presença de protrusão do núcleo pulposo (NP) através do ânulo fibroso (AF); e ruptura das fibras do ânulo fibroso, através do registro de respostas diretas positivas ("presente") ou negativas ("ausente").

O processo de avaliação ocorreu pela análise em microscópio óptico (Olympus ${ }^{\circledR}$ BX50) inicialmente com aumento de 200x, observando-se os aspectos morfológicos gerais do DIV, passando-se para 400x e 1000x, permitindo a melhor avaliação das fibras do AF, suas relações com o NP e a eventual presença de vasos sanguíneos.

Cada uma das lâminas de DIV coradas pelo método HE foi analisada quanto aos critérios pré-estabelecidos por dois pesquisadores independentes - sem o conhecimento prévio da condição do DIV (experimental ou controle). Caso não houvesse concordância na avaliação de alguma peça, um terceiro avaliador (médico patologista) revisava a lâmina, prevalecendo a sua análise como definitiva.

Achados relevantes sugestivos de degeneração e/ou característicos de DIV anormais (exemplos: ossificação intradiscal, presença de células inflamatórias), mesmo não sendo contemplados como critérios de classificação, foram discriminados na análise histológica.

A presença de um ou mais dos três critérios histológicos de degeneração classificava o DIV como "degenerado". Na ausência dos três critérios, o DIV era considerado "não-degenerado".

Após as avaliações, os resultados foram compilados em tabelas, separando-se cada um dos critérios analisados e também os classificando na condição de degenerado ou não-degenerado. Desta forma, possibilitou-se a determinação do número de discos degenerados e não-degenerados, além da determinação dos valores de concordância de cada um dos critérios analisados pelos avaliadores.

\section{RESULTADOS}

Ao fim da eutanásia, dos processos de retirada da coluna vertebral, corte manual dos blocos, amostragem do material e corte histológico, obteve-se, somados todos os animais, 60 DIV viáveis para avaliação histológica de degeneração. Deste total, 25 peças eram de discos intervertebrais submetidos à degeneração e 35 peças de DIV controle (Figura 1).

Todos os critérios de degeneração foram identificados no estudo histológico dos discos submetidos ao processo de degeneração experimental, com alto grau de concordância inter-observador.

A presença de vaso sanguíneo foi observada em 18 dos 25 DIV degenerados, com concordância de Kappa $=0,95(p<0,001)$

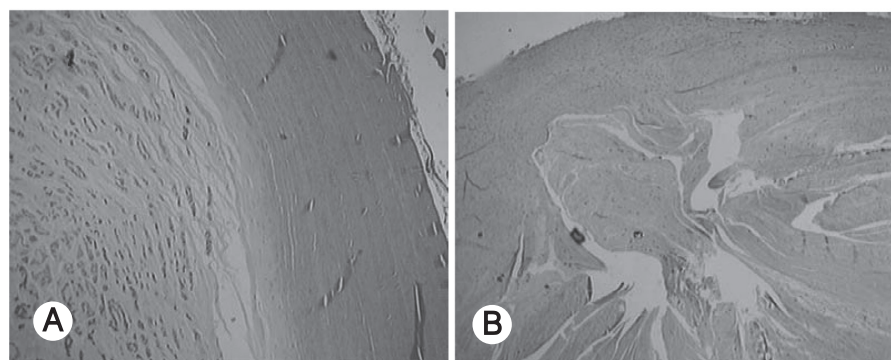

Figura 1. Lâminas coradas por HE, magnificação 200x. A) DIV Controle com Ânulo Fibroso intacto. B) DIV Experimental com desorganização lamelar e extrusão do Núcleo Pulposo.

Fonte: Laboratório de Anatomia Patológica - PUC-PR.

entre os observadores, apresentando Sensibilidade $=97 \%$ e Especificidade $=82 \%$. Em apenas um disco não-degenerado (controle) foi identificada presença de vaso sanguíneo, considerado falso-positivo.

A presença da extrusão do núcleo pulposo foi identificada em 19 dos 25 DIV experimentais, com concordância de Kappa $=0,78$ $(p<0,001)$ inter-observadores, apresentando Sensibilidade $=100 \%$ e Especificidade $=85 \%$. Não houve identificação de núcleo pulposo através do ânulo fibroso em nenhuma das 35 peças de DIV controle.

Finalmente, com relação à ruptura das fibras do $A F$, pode-se identificar a positividade em 24 dos 25 DIV degenerados, com concordância entre os observadores de Kappa =0,65 $(p<0,001)$, Sensibilidade $=88 \%$ e Especificidade $=95 \%$. Dos 35 DIV controle, observou-se ruptura das fibras do AF em sete (11\% de falso-positivos).

De acordo com a incidência do corte para amostragem do material e para o preparo histológico, em algumas lâminas, foi possível verificar o ponto correspondente à punção cirúrgica para indução de degeneração discal. Nesses locais, houve evidente extrusão do NP, com grandes alterações concomitantes nas áreas adjacentes, tais como neoformação de vasos sanguíneos e significativa ruptura das fibras do AF (Figura 2).

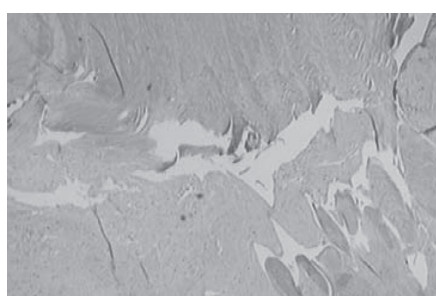

A

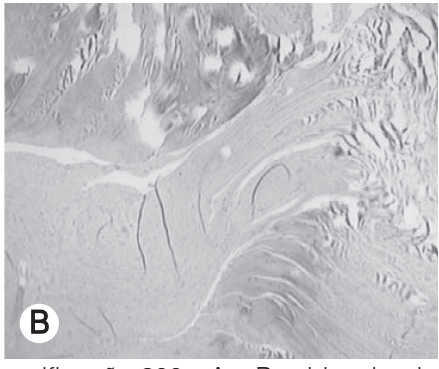

Figura 2. Lâminas coradas por HE, magnificação 200x. A e B evidenciando extrusão do NP e ruptura das fibras do AF de DIV experimentais. Fonte: Laboratório de Anatomia Patológica - PUC-PR.

Mesmo não sendo consideradas como critérios de degeneração, outras alterações foram encontradas em DIV degenerados. Em algumas amostras, verificou-se grande desorganização estrutural do NP, havendo alteração do aspecto límpido da matriz extracelular, aparente diminuição da quantidade de cordócitos e presença de agrupamentos celulares anômalos. Ainda, puderam-se identificar características histológicas típicas de reação inflamatória crônica, observando-se tecido necrótico intercalado à estrutura normal do DIV, com alteração morfológica das suas células e intensa neovascularização inicial. Outro aspecto verificado correspondeu ao processo de ossificação local, havendo tecido ósseo em formação inicial - pequena quantidade de canais de Havers - em substituição ao tecido cartilaginoso característico do DIV não-degenerado.

Utilizando-se a interpretação de Landis ${ }^{18}$ para os valores de Kappa, fica evidente que a avaliação histológica de degeneração discal através dos critérios adotados apresentou alto índice de concordância entre os observadores do estudo (Tabela 1).

Tabela 1. Concordância histológica para modelo de degeneração discal em coelhos.

\begin{tabular}{c|c|c}
\hline Critérios & Teste Kappa & Concordância \\
\hline Presença de vaso sanguíneo & 0.95 & Quase Perfeita \\
\hline Extrusão do núcleo pulposo & 0.78 & Substancial \\
\hline Rupturas de fibras do ânulo fibroso & 0.65 & Substancial \\
\hline
\end{tabular}

Fonte: Laboratório de Lesões Medulares e Trauma Experimental, SOT HUC PUCPR 
Todos os critérios apresentaram correlação confiável para determinação da presença de degeneração do disco intervertebral, com valor estatístico relevante.

\section{DISCUSSÃO}

O uso de estudos in vivo (modelos animais), tem a principal vantagem de determinar como os fatores de risco constitutivos, ambientais ou biomecânicos podem iniciar, promover, ou ainda regular as alterações discais ${ }^{19}$. Em contrapartida, modelos in vitro impedem a análise do processo de degeneração como um evento complexo, envolvendo diversas estruturas que atuam de forma inter-relacionada e que exercem papéis fundamentais no resultado final, sendo úteis apenas para identificação de eventos de curta duração 20 .

Neste estudo, a punção dos discos intervertebrais de coelhos com agulha $18 \mathrm{G}$ com profundidade limitada $(5 \mathrm{~mm})$ apresentou alterações histológicas evidentes, compatíveis com as alterações observadas na degeneração discal intervertebral ocorrida em humanos ${ }^{4,21-23}$.

Os três critérios histológicos utilizados para determinar a presença de degeneração discal neste estudo demonstraram alta prevalência entre o grupo experimental e elevado índice de concordância entre os pesquisadores. O Teste Kappa é uma medida de consenso entre observadores e mede o grau de concordância além do que seria esperado tão somente pelo acaso. Esta medida de concordância tem valores que variam de 0 a 1 , sendo que " 0 " representa não haver concordância além do puro acaso, e "1" representa a concordância perfeita ${ }^{24}$.

A ruptura das fibras do ânulo fibroso já foi observada no estudo de Boos et al ${ }^{8}$. Nesse artigo, os autores ainda analisaram aspectos como presença de proliferação celular, degeneração mucosa, morte celular, alterações granulosas, extrusão e fissuras em DIV de cadáveres e de fragmentos cirúrgicos, tendo encontrado concordância inter-observadores variando entre 80 e 96\%, com Kappa = 0,707. Já no artigo de Hoogendoorn et al25, a ruptura de fibras do AF foi um dos critérios analisados na degeneração experimental do DIV de

\section{REFERÊNCIAS}

1. Nerlich AG, Schleicher ED, Boos N. 1997 Volvo Award winner in basic science studies. Immunohistologic markers for age-related changes of human lumbar intervertebral discs. Spine (Phila Pa 1976). 1997;22(24):2781-95.

2. Roberts S, Evans H, Trivedi J, Menage J. Histology and pathology of the human intervertebral disc. J Bone Joint Surg Am. 2006;88(Suppl 2):10-4

3. Larson JW 3rd, Levicoff EA, Gilbertson LG, Kang JD. Biologic modification of animal models of intervertebral disc degeneration. J Bone Joint Surg Am. 2006;88(Suppl 2):83-7.

4. Johnson WE, Eisenstein SM, Roberts $\mathrm{S}$. Cell cluster formation in degenerate lumbar intervertebral discs is associated with increased disc cell proliferation. Connect Tissue Res. 2001;42(3):197-207.

5. Kauppila LI. Ingrowth of blood vessels in disc degeneration. Angiographic and histological studies of cadaveric spines. J Bone Joint Surg Am. 1995;77(1):26-31.

6. Kang JD, Georgescu HI, Mclntyre-Larkin L, Stefanovic-Racic M, Donaldson WF 3rd, Evans $\mathrm{CH}$. Herniated lumbar intervertebral discs spontaneously produce matrix metalloproteinases, nitric oxide, interleukin-6, and prostaglandin E2. Spine (Phila Pa 1976). 1996;21(3):271-7.

7. Brisby H. Pathology and possible mechanisms of nervous system response to disc degeneration. J Bone Joint Surg Am. 2006;88(Suppl 2):68-71.

8. Boos N, Weissbach S, Rohrbach H, Weiler C, Spratt KF, Nerlich AG. Classification of age-related changes in lumbar intervertebral discs: 2002 Volvo Award in basic science. Spine (Phila Pa 1976). 2002;27(23):2631-44

9. Andersson GB. Epidemiological features of chronic low-back pain. Lancet. 1999;354(9178):581-5.

10. Taylor VM, Deyo RA, Cherkin DC, KreuterW. Low back pain hospitalization. Recent United States trends and regional variations. Spine (Phila Pa 1976). 1994;19(11): 1207-12.

11. Hart LG, Deyo RA, Cherkin DC. Physician office visits for low back pain. Frequency, clinical evaluation, and treatment patterns from a U.S. national survey. Spine (Phila Pa 1976). 1995;20(1):11-9.

12. Katz JN. Lumbar disc disorders and low-back pain: socioeconomic factors and consequences. J Bone Joint Surg Am. 2006;(88 Suppl 2):21-4.

13. Kim KS, Yoon ST, Li J, Park JS, Hutton WC. Disc degeneration in the rabbit: a biochemical and radiological comparison between four disc injury models. Spine (Phila Pa 1976). 2005;30(1):33-7. cabras, apresentando concordância inter-observadores de Kappa $=0,54$. Ambos os estudos mostram resultados semelhantes ao observado neste trabalho (ruptura das fibras do AF com concordância inter-observador Kappa $=0,65$ ), fortalecendo a relevância deste critério para a determinação histológica de degeneração discal.

Com relação à presença de vaso sanguíneo na DDD, Kauppila ${ }^{5}$ avaliou DIV de cadáveres através de angiografia e identificou vasos entre a vértebra e o disco, com correlação estatisticamente positiva entre a o aumento da vascularização do ânulo fibroso e a degeneração discal ( $p<0.001)$.

Dos 35 DIV controle analisados neste estudo, sete apresentaram resposta positiva para ruptura de fibras do AF. Os autores atribuem este resultado à lesão que ocorre no disco durante o preparo histológico das peças com o micrótomo, levando a artefatos de técnica que podem gerar resultados falso-positivos. Por esta razão, a Sensibilidade deste critério (88\%), embora ainda seja relevante, diferiu do resultado obtido pela presença de vaso sanguíneo (97\%) e extrusão do NP (100\%), sendo refletida pelo menor dos três índices de Kappa $(0,65)$ entre os observadores. Ainda assim, a concordância substancial do teste e a alta Especificidade da presença de ruptura do AF (95\%) garantem a validade deste critério para determinação histológica da degeneração discal.

Baseando-se na análise estatística deste estudo e nos resultados semelhantes encontrados na literatura, todos os três critérios adotados têm papel importante na avaliação histológica da degeneração discal em coelhos, podendo-se afirmar com alto grau de relevância estatística que a positividade de apenas um dos critérios já é capaz de definir o disco experimental como degenerado.

\section{CONCLUSÃO}

O modelo de avaliação histológica da degeneração discal em coelhos proposto neste estudo mostrou-se viável, com alterações histológicas evidentes pós-operatórias, validando a possibilidade de indução de degeneração discal neste modelo animal para futuros estudos e comprovando o papel da histologia com alto grau de concordância inter-observadores na identificação da degeneração discal.

14. Kroeber MW, Unglaub F, Wang H, Schmid C, Thomsen M, Nerlich A, et al. New in vivo animal model to create intervertebral disc degeneration and to investigate the effects of therapeutic strategies to stimulate disc regeneration. Spine (Phila Pa 1976). 2002;27(23):2684-90.

15. Masuda $\mathrm{K}$, Aota $\mathrm{Y}$, Muehleman $\mathrm{C}$, Imai $\mathrm{Y}$, Okuma M, Thonar EJ, et al. A novel rabbit model of mild, reproducible disc degeneration by an annulus needle puncture: correlation between the degree of disc injury and radiological and histological appearances of disc degeneration. Spine (Phila Pa 1976). 2005;30(1):5-14.

16. Rousseau MA, Ulrich JA, Bass EC, Rodriguez AG, Liu JJ, Lotz JC. Stab incision for inducing intervertebral disc degeneration in the rat. Spine (Phila Pa 1976). 2007;32(1):17-24. 17.

17. Vialle $E$, Vialle $L R$, Arruda $A O$, Riet RN, Krieger $A B Q$. Análise radiológica da degeneração discal experimental em coelhos. Rev Bras Ortop. 2009;44(4):313-9.

18. Landis JR, Koch GG. The measurement of observer agreement for categorical data. Biometrics. $1977 ; 33(1): 159-74$.

19. Pritzker KP. Animal models for osteoarthritis: processes, problems and prospects. Ann Rheum Dis. 1994;53(6):406-20.

20. Goel VK, Panjabi MM, Patwardhan AG, Dooris AP, Serhan H; American Society for Testing and Materials. Test protocols for evaluation of spinal implants. J Bone Joint Surg Am. 2006;88(Suppl 2):103-9.

21. An HS, Masuda K. Relevance of in vitro and in vivo models for intervertebral disc degeneration. J Bone Joint Surg Am. 2006;88(Suppl 2):88-94.

22. Battié MC, Videman T. Lumbar disc degeneration: epidemiology and genetics. J Bone Joint Surg Am. 2006;(88Suppl 2):3-9.

23. Sobajima S, Kompel JF, Kim JS, Wallach CJ, Robertson DD, Vogt MT, et al. A slowly progressive and reproducible animal model of intervertebral disc degeneration characterized by MRI, X-ray, and histology. Spine (Phila Pa 1976). 2005;30(1):15-24.

24. Cohen J. A coefficient of agreement for nominal scales. Educ Psychol Meas. 1960;20(1):37-46.

25. Hoogendoorn RJ, Wuisman PI, SmitTH, Everts VE, Helder MN. Experimental intervertebral disc degeneration induced by chondroitinase $A B C$ in the goat. Spine (Phila Pa 1976). 2007;32(17):1816-25. 